\title{
RECENT DEVELOPMENTS IN ASTRONOMY IN VIETNAM
}

\author{
NGUYEN QUANG RIEU \\ Observatoire de Paris, Paris, France
}

\section{Survey of the Situation}

Astronomy in Vietnam is still heavily underdeveloped. The few telescopes of the Observatory of Phu Lien (Hai Phong) were moved during the wars and become unusable. Most of the astronomers who graduated from the universities in the former Soviet Union are now working in other fields. Astronomy and astrophysics are among the basic sciences that Vietnam is willing to promote.

The total solar eclipse on October 24, 1995, in the south of Vietnam was an opportunity to give a boost to astronomy. On this occasion, the country received substantial scientific assistance from the International Astronomical Union (IAU) and other foreign institutions. A National Conference co-sponsored by the IAU, the French Ministry of Foreign Affairs (MFA), the French National Centre of Scientific Research (CNRS), and the Observatory of Paris was organized in Ho Chi Minh City in October 1995 by the Ministry of Sciences, Technology and Environment. A polar siderostat, a small two-antenna radio interferometer operating around $5000 \mathrm{MHz}$, built at the Observatory of Paris, and a few small optical telescopes were given to the National University of Vietnam in Hanoi by the Observatory of Paris with the financial support of the MFA. These instruments were used to observe the solar eclipse of October 1995 at Phan Thiet in south Vietnam. A 45-cm optical telescope and a 10-cm solar telescope were donated by the Observatory of Tokyo to the University of Pedagogy in Hanoi.

\section{Teaching Tasks}

The mostt urgent action is to educate a new generation of Vietnamese astronomers. Basic elementary notions of astronomy are taught at the Pedagogical University of Hanoi, the University of Vinh (central Vietnam) and the University of Ho Chi Minh City. Modern astrophysics is practically absent from the courses. A personal computer and a number of textbooks in astrophysics, written in English and Vietnamese, were offered by the IAU to the University of Vinh. A first series of introductory lectures in astrophysics was given at the National University of Hanoi in October 1995 by foreign astronomers. The IAU has accepted a project to enhance astronomy in Vietnam through a TAD (Teaching for Astronomy Development) programme. The first TAD school in Vietnam will be held at the University of Vinh in September 1- 14, 1997. The audience will be the teachers in astronomy and physics students from all over Vietnam. The faculty members will be Profs. Donat G. Wentzel and Nguyen Quang Rieu. The main topics are the solar system, stellar evolution, the interstellar medium and some other subjects related to radio astronomy.

\section{Collaborative Research Programmes}

There exist collaborative research programmes with foreign observatories. A research fellow from the Laser Institute in Hanoi is working in the Department of Millimeter Radio Astronomy of the Observatory of Paris in the field of molecular spectroscopy. A graduate student of the National University of Hanoi is finishing his Ph.D. thesis, at the same institute in Paris, on molecular emission from galaxies and on image processing. Both have French scholarships from the National Research Centre and the Ministry of Foreign Affairs. They will form with students trainiing in other countries a nucleus of research fellows in astronomy and astrophysics in Vietnam. 
An agreement for collaboration between the University Pierre and Marie Curie in Paris and the Observatory of Paris, on the one hand, and the National University of Vietnam, on the other, is being negotiated. The aim is mainly to train teachers in astronomy and in some aspects of radio physics related to astronomy.

\section{Planetarium}

A project to build a planetarium with an 8-m dome in Vinh is underway with the assistance of Japan. A more ambitious project to install a $15-\mathrm{m}$ planetarium in Hanoi is under consideration in collaboration with France. The science of the universe promoted on a large scale should not only provide scientific knowledge to the public, but should also help to combat superstition which is rampant in the country.

\section{SUMMARY OF DISCUSSION OF MORNING SESSIONS (Onuora to Haubold)}

In discussion of Onuora's paper, parallels were drawn between the situations in India and in Nigeria. Indian universities were also going through a "resource crunch". Some astronomers, however, are influential with the government and they are the ones who could convince politicians to invest in astronomy. It was pointed out, however, that governments change. Japanese astronomers had been willing to help one Nigerian astronomer with a project, but after a change of government the project ceased to be feasible. In reply to a direct question, Onuora said that the only arguments for astronomy that carried any weight with Nigerian politicians were those based on the technological spin-offs that astronomy can produce. It was pointed out that amateur astronomers who are businessmen or well-placed in other professions can be useful allies. In respect of Othman's paper it was emphasized that even when sponsors are found, they need to be educated about the nature and goals of astronomy.

In both Othman's and Rieu's papers (the latter read by Wentzel) the role of astronomy in combatting superstition was discussed. Some interpreted "superstition" as astrology and had found it impossible to explain the cause of eclipses without first attacking the astrological beliefs held by many of their students. Others interpreted the term "superstition" more widely and warned against attacking local beliefs in a way that would only strengthen them. It is better to present scientific ideas as alternative ways of looking at otherwise inexplicable phenomena.

After Kochhar's references to the cost of publications, it was commented that the IAU's change of publisher would result in a decrease in the cost of publications by a factor of two. Moreover, the IAU does send all its publications free of charge to many developing countries.

After Bochkarev's paper it was asked whether or not it would be useful for the IAU to recommend a percentage of GDP that should be devoted to scientific research and a percentage of that amount that should be devoted to astronomy. It was pointed out that comparing different countries' scientific expenditure as fractions of GDP could be misleading: some countries include all support of R \& D in such percentages, others only government support. The IAU should be very cautious in making such recommendations and could best work through ICSU.

On Wentzel's paper, it was commented that VLPs had been useful, but there must be jobs available in the countries where the programmes are run, and possibilities for the students concerned to continue studies abroad. The personal preferences of professors sometimes played too large a role in who received such opportunities. It was said that Bart Bol had been an inspiring teacher aat the Bandung ISYA, and it was desirable that someone of that calibre should be at each ISYA, for the whole period of the school.

Jørgensen was asked how many travel grant applications had been rejected by Commission 38 . He replied, very few. As long as an application was within the guidelines and supported by the applicant's home and host institutions, it was nearly always accepted. The Commission did not act like a granting council and assess the project in detail. The possibility of an IAU role in the UN/ESA Workshops was mentioned.

Note: The paper by M.C. Pineda de Carias was read by title only. 